\title{
Amigdalectomía y adenoidectomía: Conceptos, técnicas y recomendaciones
}

\author{
Tonsillectomy and adenoidectomy: Concepts, techniques and recommendations \\ Andrés Alvo $\mathbf{V}^{1}$, Marcel Sauvalle $\mathbf{C}^{1}$, Cecilia Sedano $\mathbf{M}^{2}$, Romina Gianini $\mathbf{V}^{3}$.
}

\begin{abstract}
RESUMEN
La cirugía de adenoides y amígdalas es de los procedimientos más frecuentemente realizados dentro de la especialidad, y de las operaciones más comunes en niños. Actualmente, la indicación más común es la hiperplasia asociada a trastornos del sueño, desplazando a las infecciones recurrentes como primera causa de cirugía. Debido a esto, es importante conocer los aspectos relevantes en cuanto a anatomía, fisiología, indicaciones, técnica quirúrgica y complicaciones del procedimiento.

Esta revisión pretende presentar el estado del arte actual y entregar una guía inicial a los especialistas en formación, así como una actualización para quienes ya poseen experiencia dentro de la otorrinolaringología.
\end{abstract}

Palabras clave: Adenoidectomía, amigdalectomía, faringe.

\begin{abstract}
Surgery of the adenoids and tonsils is among the most frequently performed procedures within the speciality, and one of the commonest surgeries in children. Currently, the most frequent indication is sleep disorder-associated hyperplasia, displacing recurrent infections as the leading cause of surgery. Because of this, it is important to know relevant issues about anatomy, physiology, indications, operative technique and complications of the procedure.

The intention of this review is to present the current state-of-the-art and to provide an initial guide for specialists in formation, as well as an update for experienced otorhinolaryngologists.
\end{abstract}

Key words: Adenoidectomy, tonsillectomy, pharynx.

\footnotetext{
Médico, Servicio de Otorrinolaringología Hospital Clínico Universidad de Chile.

2 Medico, Servicio de Otorrinolaringología Hospital Barros Luco-Trudeau.

3 Interna de Medicina, Universidad de Chile.
}

Recibido el 7 de septiembre de 2015. Aceptado el 29 de octubre de 2015. 


\section{ANATOMÍA}

El anillo linfático faríngeo, antiguamente denominado anillo de Waldayer, corresponde al conjunto de estructuras linfoides ubicadas desde la nasofaringe hasta la base de la lengua. Está formado por las tonsilas faríngeas (adenoides), tonsilas peritubáricas, cordones faríngeos laterales, tonsilas palatinas y tonsila lingual. Participan en la inmunidad local y la vigilancia inmunológica. Producen inmunoglobulinas, IgA secretoria por parte del adenoides e IgA, IgM, IgG, $\lg \mathrm{D}$ e $\lg E$ por parte de las amígdalas ${ }^{1}$. Debido a su epitelio especializado son importantes presentadores y procesadores de antígenos.

Las amígdalas 0 tonsilas palatinas corresponden a dos estructuras que se ubican en la fosa tonsilar, delimitada por los pilares palatogloso hacia anterior, palatofaríngeo hacia posterior, músculo constrictor superior de la faringe hacia lateral, paladar blando hacia superior y raíz de la lengua como limite inferior. Están rodeadas por un tejido de mayor densidad denominado cápsula amigdalina, dependiente de la fascia faringobasilar. Entre estas estructuras queda delimitado el espacio periamigdalino, donde se encuentran algunas glándulas salivales menores conocidas como glándulas de Weber, cuya infección tendría relación con la etiopatogenia de los abscesos periamigdalinos $^{2}$. La cara medial de las tonsilas palatinas es mamelonada, y posee la desembocadura de las criptas amigdalinas. Su irrigación se divide en 3 segmentos: el segmento superior se encuentra irrigado por ramas de la arteria palatina descendente, su tercio medio por la arteria faríngea ascendente y su tercio inferior por ramas tonsilares de las arterias lingual, facial y palatina ascendente. Su inervación depende principalmente del nervio glosofaríngeo (IX par).

La amígdala faríngea, comúnmente conocida como adenoides, corresponde a una estructura linfoide que en conjunto con las amígdalas palatinas forman las estructuras más importantes del anillo linfático faríngeo. Se ubica en la pared posterosuperior de la nasofaringe. Una de sus características es su crecimiento progresivo durante la infancia, alcanzando su máximo tamaño alrededor de los 7 años en promedio, para luego involucionar hacia la pubertad. Su irrigación depende de las arterias palatina ascendente (rama de la arteria facial), faríngea ascendente (rama de la arteria carótida externa) y cervical ascendente (rama del tronco tirocervical). La inervación está dada principalmente por los nervios glosofaríngeo (IX par) y vago (X par).

\section{AMIGDALECTOMÍA}

La técnica quirúrgica clásica es la amigdalectomía extracapsular bilateral, que corresponde a la resección quirúrgica de ambas amígdalas palatinas junto a su cápsula, mediante la disección del espacio periamigdalino. Corresponde a uno de los procedimientos quirúrgicos realizados con mayor frecuencia por los otorrinolaringólogos, alcanzando en Estados Unidos más de 500.000 procedimientos anuales en menores de 15 años ${ }^{3-5}$.

Las indicaciones y contraindicaciones de este procedimiento se resumen en la Tabla 16-8.

Tabla 1. Indicaciones y contraindicaciones de amigdalectomía

\begin{tabular}{|lll|}
\hline Indicaciones absolutas & Indicaciones relativas ${ }^{9}$ & Contraindicaciones relativas \\
\hline $\begin{array}{l}\text { Roncopatía con pausas o SAHOS (atribuible } \\
\text { a hiperplasia amigdalina grado III ó IV) }\end{array}$ & Enfermedad de Berger (Nefropatía por IgA) & Trastornos de la coagulación \\
$\begin{array}{l}\text { Amigdalitis a repetición (definida } \\
\text { según criterios de Paradise), o crónica }\end{array}$ & Síndrome PANDAS & Asma no controlada \\
Posterior a $2^{\circ}$ absceso periamigdalino & Síndrome PFAPA & \\
Amigdalitis hemorrágica & Tonsilolitiasis & Proceso infeccioso concomitante \\
& & Riesgo de insuficiencia \\
Asimetría amigdalina & Otros más discutidos: Hiperplasia asociada a & velofaríngea \\
(con sospecha de cáncer) & trastornos craneofaciales, dentales y de la & \\
& deglución; pustulosis palmoplantar/psoriasis; & \\
& Eritema nodoso; portadores de SBHGA y difteria, etc. & \\
\hline
\end{tabular}

*SAHOS: Síndrome de apnea/hipopnea obstructiva del sueño

${ }^{*}$ Criterios de Paradise: 7 episodios de amigdalitis bacteriana en 1 año, 5 anuales por 2 años consecutivos, 03 anuales en 3 años consecutivos ${ }^{8}$. 


\section{Técnica quirúrgica ${ }^{3,5,10}$}

Existen diversos métodos para realizar una amigdalectomía y principalmente se clasifican en fríos y calientes. Los métodos fríos corresponden a aquéllos que no liberan energía térmica durante el procedimiento, lo que teóricamente podría disminuir el dolor posoperatorio. Los métodos calientes liberan distintas cantidades de energía térmica que produce un efecto cauterizador, potencialmente disminuyendo tanto el tiempo quirúrgico como el sangrado intraoperatorio. A pesar de lo anterior, no existe una clara evidencia a través de estudios randomizados que logre determinar qué método quirúrgico es más seguro en términos de sangrado y dolor posoperatorio; la mayor diferencia entre las distintas técnicas son los costos de los instrumentos utilizados ${ }^{5}$.

Estos procedimientos también se pueden clasificar en extracapsulares o intracapsulares según la preservación de la cápsula tonsilar ${ }^{5}$.

1. Amigdalectomía extracapsular. Dentro de los instrumentos más frecuentemente utilizados se encuentran ${ }^{10}$ :

a. Disección fría: Corresponde a la amigdalectomía total realizada con bisturí frio y/o tijeras. Al igual que otras técnicas se realiza una incisión en el polo superior y mediante una tracción medial mantenida de la amígdala, se va disecando por el plano avascular del espacio periamigdalino, desde superior a inferior ${ }^{5}$. Gracias a la tracción medial, se evita lesionar la musculatura faríngea.

La hemostasia se puede realizar con puntos hemostáticos, packing o tórulas embebidas en subgalato de bismuto, técnica hemostática que mencionaremos más adelante.

b. Asa: Tipo de disección fría y rápida en la que se extrae la amígdala con asa de alambre ${ }^{11}$. Se debe traccionar el polo superior de la amígdala hacia medial, rodear con el asa y luego realizar un movimiento firme y descendente entre ambos pilares musculares hasta extraerla completamente. Muchas veces es necesario realizar una disección fría para exponer adecuadamente la amígdala y luego utilizar el asa para completar la resección del pedículo. La hemostasia debe ser cuidadosa, ya sea con compresas, suturas reabsorbibles, electrocauterización y/o subgalato de bismuto. c. Daniels: Corresponde a la amigdalectomía realizada con guillotina. Tiene la ventaja de ser rápida y precisa, sin embargo se debe tener especial cuidado con el sangrado tanto intra como posoperatorio y la lesión de la musculatura faríngea. Sus defensores argumentan que tendría el beneficio teórico de comprimir y colapsar los vasos antes de la disección.

d. Electrocauterización: La corriente eléctrica de un generador pasa a través del tejido entre dos electrodos y el calor generado, que va de los 400 a los $600^{\circ} \mathrm{C}$, corta el tejido y sella los vasos sanguíneos. Esta puede ser monopolar si la corriente pasa del instrumento quirúrgico, a través del paciente, a un electrodo ubicado en su pierna o bipolar si la corriente pasa a través del tejido, entre dos electrodos localizados en las puntas de un fórceps 0 una tijera. Es la técnica más común en todo el mundo.

e. Bisturí armónico: Corresponde a un bisturí que utiliza energía ultrasónica para vibrar a 55.000 ciclos por segundo. La vibración transfiere energía mecánica al tejido que permite cortar y coagular al mismo tiempo.

f. Ablación por radiofrecuencia (Coblator): Corresponde a un instrumento que utiliza la energía de la radiofrecuencia para excitar electrolitos en un medio conductor, tal como una solución salina, creando un plasma enfocado con precisión. Las partículas energizadas del plasma tienen suficiente energía como para romper los lazos moleculares del tejido, provocando que el tejido se disuelva a temperaturas relativamente bajas. El resultado es la remoción del tejido en cuestión con un daño mínimo a los tejidos circundantes. También permite realizar hemostasia, coagulando los vasos sangrantes.

g. Microdebridador: Se utiliza principalmente para amigdalectomía intracapsular, técnica que mencionaremos más adelante. Corresponde a un instrumento que posee un sistema rotacional distal asociado a succión que permite disecar estructuras con mínimo trauma a los tejidos circundantes.

\section{Amigdalectomía intracapsular:}

Corresponde a una amigdalectomía subtotal en la que se preserva la cápsula tonsilar. La amígdala se extrae por piezas conservando la cápsula, incluso algunos dejan parte del tejido amigdalino. 
Se realiza principalmente con microdebridador y radiofrecuencia. Podría estar indicada cuando el tamaño tonsilar es el causante de los síntomas; sin embargo no tiene indicación en casos de amigdalitis recurrente u otros cuadros infecciosos. La ventaja es que potencialmente disminuiría el dolor y el sangrado posoperatorio, aunque esto es materia de debate ${ }^{5,12}$. Por otro lado, existe el riesgo de reaparición de la sintomatología por nuevo crecimiento del remanente amigdalino.

\section{ADENOIDECTOMÍA}

La adenoidectomía corresponde a la extracción quirúrgica del tejido adenoideo en la nasofaringe. En la mayoría de los casos, esta cirugía cumple un doble objetivo: mecánico (eliminar la obstrucción a nivel de la faringe) y biológico (eliminar el tejido alterado por la inflamación y/o infección crónica) $)^{6}$. Es un procedimiento principalmente pediátrico debido a su frecuente involución hacia la pubertad. En general se tiende a evitar antes de los 12-14 meses de edad, aunque no es una contraindicación absoluta y debe evaluarse caso a caso. De estar indicados ambos procedimientos, habitualmente se realiza en el mismo tiempo quirúrgico que la amigdalectomía. Sus indicaciones y contraindicaciones se resumen en la Tabla $2^{6,7}$.

\section{Técnica quirúrgica ${ }^{3}$}

Al igual que con la amigdalectomía, existen diversas técnicas para extraer la tonsila faríngea 0 adenoides. Dentro de las más utilizadas encontramos:

a. Extracción con cureta: El adenótomo es un instrumento metálico con forma de cuchara que permite realizar la adenoidectomia mediante raspado nasofaríngeo. Previo al procedimiento se elige la cureta según la inspección del adenoides y se inserta mediante palpación, visión indirecta con la ayuda de un espejo laríngeo 0 directa mediante elevación del paladar blando, ubicándose superior al vómer septal, contra la región posterosuperior de la nasofaringe. Se realiza un barrido descendente para remover todo el tejido, teniendo especial cuidado para evitar dañar la musculatura faríngea. Se puede elegir una segunda cureta de menor tamaño para extraer los restos. La hemostasia se puede realizar con compresión mecánica 0 packing, oximetazolina 0 tórulas embebidas en subgalato de bismuto por 5 minutos hasta detener el sangrado.

b. Coagulación-succión: Técnica en la que se utiliza un instrumento cilíndrico de 10 French, que posee un sistema de succión central asociado a un sistema de coagulación periférico. Se

Tabla 2. Indicaciones y contraindicaciones de adenoidectomía

\begin{tabular}{|ll|}
\hline Indicaciones & Contraindicaciones relativas \\
\hline $\begin{array}{l}\text { Roncopatía con pausas o SAHOS } \\
\text { (atribuible a hiperplasia adenoidea grado II ó III) }\end{array}$ & Trastornos de la coagulación \\
Otitis media aguda recurrente & $\begin{array}{l}\text { Fisuras palatinas y otros factores de riesgo para insuficiencia } \\
\text { velofaríngea }\end{array}$ \\
Adenoiditis crónica & Fiebre o cuadro infeccioso concomitante \\
Otitis media con efusión \\
(en especial en recidivas post-tubos de ventilación)
\end{tabular}


debe conectar al sistema monopolar y se utiliza en modo "Spray". Este instrumento se debe posicionar al centro del adenoides y aplicar corriente durante unos segundos. El tejido adenoideo se licúa mientras se va aspirando al mismo instante. No se debe realizar muy superficial, ya que el tejido no se licua, se cauteriza y forma costras.

c. Microdebridador: Corresponde a la misma técnica mencionada en amigdalectomía. Tiene la ventaja de ser una técnica rápida y muy precisa, de elección en los casos de insuficiencia velofaríngea en que conviene realizar una adenoidectomía exclusiva del polo superior, para que el polo inferior se mantenga y permita el correcto cierre velofaríngeo. Especial cuidado se debe tener con estructuras vecinas, como por ejemplo, el torustubario. Estas últimas dos técnicas se realizan habitualmente bajo visión endoscópica.

d. Láser: Técnica que podría ser utilizada para adenoidectomía. No se recomienda su uso ya que produce cicatrización excesiva del tejido nasofarínge $0^{10}$.

\section{RECOMENDACIONES PREOPERATORIAS}

Al igual que todos los procedimientos quirúrgicos se debe realizar una completa evaluación preoperatoria con énfasis en la anamnesis y el examen físico, que nos permitan pesquisar y manejar a tiempo patologías concomitantes que podrían alterar la correcta evolución tanto intra como posoperatoria del paciente ${ }^{3,6}$

- Anamnesis: Se debe realizar una completa anamnesis en todos los casos con énfasis en antecedentes médicos, quirúrgicos, familiares y alergias. Es importante registrar los medicamentos que esté utilizando el paciente, para prevenir interacciones y complicaciones secundarias al consumo de éstos.

- Examen físico: Se debe realizar examen físico completo y medición de signos vitales, sobre todo en niños. Énfasis en alteraciones cardiopulmonares, estigmas de coagulopatías, síndromes neuromusculares, etc. Es importante realizar examen velofaríngeo para determinar la predisposición a insuficiencia velofaríngea que se exacerbe tras la adenoidectomía ${ }^{3}$. Los factores de riesgo para esta alteración son los siguientes: Historia de regurgitación, anormalidades del paladar (fisuras palatinas evidentes, escotadura en paladar duro, diástasis muscular), paladar corto y alteraciones de la úvula (bífida, ausente). Es importante descartar la presencia de un cuadro infeccioso concomitante, ya que al ser un procedimiento electivo es indicación de postergación de la cirugía.

- Exámenes preoperatorios: Aunque su real utilidad es materia de debate, usualmente se solicitan algunos exámenes de laboratorio de rutina en todos los pacientes. En caso de sospecha clínica o resultados alterados se pueden agregando pruebas específicas para ciertas patologías.

a. Exámenes de laboratorio: Habitualmente solicitados a todas las personas previo a una intervención quirúrgica. Permiten detectar alteraciones como anemia, trombocitopenia, trastornos de la coagulación, etc. y prevenir posibles complicaciones. Dentro de la batería inicial se encuentran: hemograma, recuento de plaquetas, tiempo de protrombina y tiempo de trombina parcial activada (TTPA $)^{5}$. En caso de antecedente de enfermedades cardiovasculares se debe incluir electrocardiograma y eventualmente radiografía de tórax.

b. Nasofibroscopía: Examen endoscópico que permite determinar el tamaño del adenoides. Es mucho más sensible y específico que la radiografía de cavum. No es estrictamente necesario para realizar adenoidectomía, aunque permite además evaluar la función velofaríngea, por lo que tiene un rol importante ante la sospecha clínica de riesgo de insuficiencia previo a la cirugía.

c. Radiografía de columna cervical: Especialmente en casos de pacientes con diagnóstico de síndrome de Down, debido al riesgo de subluxación atlantoaxoídea tras la cirugía ${ }^{3}$.

d. Polisomnografía: Corresponde al gold standard para el diagnóstico y la clasificación según severidad del síndrome de apnea obstructiva del sueño ${ }^{3}$. Consiste en el 
control de variables fisiológicas durante el sueño: intercambio gaseoso, esfuerzo respiratorio, flujo aéreo, ronquidos, posición corporal, etapas del sueño, entre otros. No corresponde a un examen preoperatorio de rutina, sin embargo algunos estudios lo recomiendan en casos particulares como menores de 2 años, síndrome de apnea del sueño severo o sospecha de éste y comorbilidades como obesidad, síndrome de Down, anormalidades craneofaciales y enfermedades neuromusculares s,7,12. $^{5}$.

- Pases quirúrgicos: En caso de antecedentes médicos en tratamiento, el médico especialista a cargo debe extender un pase quirúrgico autorizando la cirugía, siempre y cuando la patología se encuentre compensada.

- Evaluación dental: Podría ser de utilidad en caso de pacientes con potencial pérdida de piezas dentales secundario a la intubación 0 al instrumental quirúrgico ${ }^{3}$.

- Evaluación preanestésica: Evaluación realizada por el anestesiólogo, de preferencia 15 días previo a la intervención. En el caso de pacientes pediátricos, deben ser acompañados por sus padres. Lo principal es evaluar comorbilidades, trastornos hemorrágicos, síndrome de apnea obstructiva del sueño, mala tolerancia a la anestesia en intervenciones previas, etc. Se debe pesar y medir al paciente para el posterior cálculo de las dosis de anestésicos a utilizar.

- Medicación prequirúrgica: Existen estudios que recomiendan una adecuada hidratación durante el preoperatorio para disminuir el dolor y molestias posoperatorias. Se debe suspender el consumo de ácido acetilsalicílico y otros antiinflamatorios no-esteroidales una semana previo a cirugía, ya que podrían aumentar las complicaciones hemorrágicas ${ }^{3}$.

- Ingreso: Los pacientes pediátricos deben ingresar al centro hospitalario a primera hora el mismo día de su intervención, habiendo completado un ayuno de sólidos y líquidos de lo menos 6 horas previo a la cirugía. Algunos sugieren que los adultos ingresen la noche anterior al procedimiento y completar el ayuno de 8 horas en el recinto.

\section{RECOMENDACIONES INTRAOPERATORIAS}

Existen varios factores que se deben tener en consideración durante el intraoperatorio. Además de la correcta técnica quirúrgica, hemostasia, anestesia y posición del paciente, existen recomendaciones que permiten potenciar la calidad y el bienestar durante el posoperatorio.

a. Dexametasona: Se recomienda el uso de dexametasona intraoperatoria, en dosis única de $0,5 \mathrm{mg} / \mathrm{kg}$ endovenoso (máximo $16 \mathrm{mg}$ ) que reduce el dolor posoperatorio y disminuye las náuseas y vómitos ${ }^{3}$.

b. Subgalato de bismuto: El subgalato de bismuto corresponde a un metal pesado, relativamente insoluble en agua, con cualidades astringentes que actuaría mediante la activación del factor de Hageman (XII) de la cascada de coagulación, acelera la formación del coágulo y mejora la hemostasia. Se utiliza en forma de pasta, a la que se puede adicionar adrenalina, participando en la hemostasia gracias a su efecto vasoconstrictor. Se debe aplicar tórulas embebidas en la pasta tanto en el lecho adenoideo como en las fosas amigdalinas durante 5 minutos. Luego, se lava el lecho, opcionalmente dejando una delgada capa en el sitio operatorio. Existe una mínima evidencia científica que apoya su uso en la adenoamigdalectomía, disminuyendo la incidencia de sangrado $0^{13}$. Algunos argumentan que el real efecto hemostático está dado por la adrenalina incluida en la mezcla, siendo el subgalato un mero medio de transporte. Los beneficios son su bajo costo, fácil utilización y baja tasa de efectos adversos, disminuyendo el riesgo de sangrado posamigdalectomía ${ }^{14}$. Además, debido a su color amarillo-verdoso, facilita la detección de puntos de sangrado en el lecho amigdalino ${ }^{15}$.

La mayoría de las revisiones descarta efectos adversos asociados a su uso. Existe el caso anecdótico de ignición amigdalina durante electrocoagulación, secundaria al uso de subgalato, sin embargo tras recreaciones de este evento, se evidencia que podrían deberse a la fuga de aire por el tubo endotraqueal en pacientes que reciban oxígeno en concentraciones mayores al $30 \%$. No se han evidenciado complicaciones tras la ingesta 
0 aspiración de subgalato de bismuto, sin embargo, según estudios en ratas, la aspiración de este compuesto podría producir cuadros de neumonitis, sobre todo en pacientes con patología pulmonar de base. Los casos de encefalopatía por subgalato de bismuto se presentan en pacientes con consumo crónico de esta sustancia, como manejo odorante de colostomías ${ }^{16}$.

c. Antibióticos: No existe evidencia que demuestre la disminución de complicaciones infecciosas postamigdalectomía. Sólo disminuye los episodios de fiebre posoperatoria, sin embargo no se recomienda su uso rutinario.

\section{RECOMENDACIONES POSOPERATORIAS}

a. Observación: Luego de la cirugía, se recomienda trasladar al paciente a una sala de recuperación, donde se mantenga en observación durante al menos 30 minutos. Se recomienda mantener el ayuno por 4-6 horas, permitiendo únicamente la ingesta de agua fría.

b. Alta hospitalaria: Para los pacientes pediátricos el alta hospitalaria se puede dar el mismo día de la intervención, al menos 6 horas posterior al término de ésta. Es importante evaluar la realimentación, diuresis y ausencia de hemorragia. En el caso de comorbilidades importantes, síndrome de apnea obstructiva del sueño severa, complicaciones posoperatorias, vivir lejos de un centro hospitalario, etc; se recomienda mantener una noche durante observación previo al alta ${ }^{3,5}$.

c. Analgesia: Se recomienda el uso de paracetamol horario por 5-7 días en dosis ajustadas según el peso del paciente. No existe evidencia significativa que demuestre que el uso de AINEs se asocie a mayor riesgo de sangrado en pacientes pediátricos (con la posible excepción del ketorolaco), es por esto que es posible adicionar ibuprofeno horario, ajustando la dosis según el peso del paciente. No se recomienda el uso de opiáceos como codeína, ya que no se ha demostrado efecto beneficioso superior al del paracetamol por sí solo y además agrega todos los efectos secundarios de los narcóticos (constipación, náuseas, vómitos y depresión respiratoria).

d. Control: El primer control debe ser realizado 1 semana posterior al procedimiento, por su médico tratante.

\section{COMPLICACIONES 3,6}

Las complicaciones de ambos procedimientos son similares y se pueden clasificar en relacionadas a la anestesia, intraoperatorias, posoperatorias precoces y posoperatorias tardías; y se detallan en la Tabla 3.

Tabla 3. Principales complicaciones de adenoidectomía y amigdalectomía

\begin{tabular}{|llll|}
\hline $\begin{array}{l}\text { Complicaciones } \\
\text { anestésicas }\end{array}$ & $\begin{array}{l}\text { Complicaciones } \\
\text { intraoperatorias }\end{array}$ & $\begin{array}{l}\text { Complicaciones posquirúrgicas } \\
\text { inmediatas }\end{array}$ & $\begin{array}{l}\text { Complicaciones } \\
\text { posquirúrgicas tardías }\end{array}$ \\
\hline Arritmias & Traumatismo o luxación dental & Hemorragia & Insuficiencia velar \\
Broncoespasmo & Luxación & Broncoaspiración \\
temporomandibular & de sangre & $\begin{array}{l}\text { Estenosis nasofaríngea } \\
\text { y/u orofaríngea }\end{array}$ \\
Laringoespasmo & Heridas velares o velofaríngeas & Infecciones & Recidiva \\
Edema pulmonar & Laceraciones de los & Heridas de úvula y velo & \\
ex - vacuo & espacios parafaríngeos & & \\
Aspiración & Lesiones vasculares y hemorragias & Parálisis de nervios lingual, \\
& & hipogloso o glosofaríngeo & \\
Accesos de tos & & & \\
Náuseas y vómitos & & & \\
\hline
\end{tabular}




\section{Complicaciones anestésicas}

Se producen en el perioperatorio secundarios principalmente a la intubación, medicamentos utilizados o dosis administradas. En caso de dosis subóptimas se pueden producir alteraciones del ritmo cardiaco o broncoespasmo. En caso de dosis mayores a las recomendadas existe el riesgo de aspiración y broncoespasmo.

Posterior a la extubación se puede producir aspiración de secreciones, laringoespasmo o laringitis subglótica. Las correspondientes al posoperatorio inmediato son accesos de tos, náuseas y vómitos, caso en que se recomienda la administración de ondansetrón, antagonista del receptor de serotonina que ha demostrado ser efectivo y puede ser considerado un antiemético de primera línea en niños sometidos a cirugía.

\section{Complicaciones intraoperatorias}

a. Traumatismo o luxaciones dentales: Secundarios a la intubación o instrumentalización quirúrgica.

b. Luxación temporomandibular: Tras la prolongada apertura bucal se puede producir luxación de la articulación temporomandibular, manifestándose como dolor e impotencia funcional.

c. Heridas velares o velofaringeas: Principalmente por instrumental quirúrgico.

d. Rotura de espacios parafaríngeos: Lesión de la musculatura faríngea. Es más frecuente en caso de procesos infecciosos, donde los planos quirúrgicos están mal definidos.

e. Hemorragias: Debido a lesiones vasculares $0 \mathrm{a}$ una técnica hemostática deficiente durante el intraoperatorio.

\section{COMPLICACIONES POSQUIRÚRGICAS}

\section{a. Inmediatas}

- Hemorragia: Es la complicación más frecuente en el posoperatorio inmediato. Ocurre entre $3 \%$ y $5 \%$ de los pacientes intervenidos y su incidencia aumenta con la edad del paciente. Noventa por ciento ocurre dentro de las primeras 8 horas posoperatorias y $10 \%$ puede ocurrir hasta el décimo día. En adenoidectomías puede ocurrir por desgarros de la mucosa faríngea, tejido adenoideo remanente, lesión de la arteria vomeriana 0 daño a la cola del cornete inferior. En amigdalectomías es usualmente secundaria a lesiones vasculares, como arteria faríngea ascendente aberrante, arteria carótida aberrante retrofaríngea posterior (se asocia a fisura palatina o fisura submucosa) o lesión del pedículo vascular amigdalino. También se puede producir hemorragia debido a una exéresis incompleta de amígdalas/adenoides $0 \mathrm{a}$ una coagulopatía subyacente no diagnosticada. En el caso de sangrados tardíos, muchas veces ocurren cuando el tejido cauterizado se altera, alrededor de la primera semana posoperatoria. En caso de persistencia a pesar de medidas generales y farmacológicas, se requiere de revisión quirúrgica. El tratamiento va desde el packing, cauterización o sutura hasta la embolización o ligadura de la carótida externa.

- Broncoaspiración de sangre: Complicación del intraoperatorio o posoperatorio inmediato. Puede llegar a producir asfixia y muerte. Es por esto que durante la cirugía se debe proteger la vía aérea con un tubo con cuffy una gasa en la hipofaringe.

- Heridas de úvula y velo del paladar: Producidas por instrumental quirúrgico.

- Infecciones: Como se mencionó anteriormente, no se encontró evidencia que los antibióticos disminuyan la morbilidad posamigdalectomía, sólo disminuyen los episodios de fiebre posoperatoria. Actualmente no se recomienda su uso.

- Parálisis de nervios lingual e hipogloso: Debido a la compresión y edema lingual secundario al instrumental de apertura bucal. Para evitarlo, se recomienda liberar la apertura bucal durante la hemostasia que se realiza entre una amígdala y la otra.

- Lesión de nervio glosofaríngeo: Complicación infrecuente que se debe al trayecto anatómico del nervio glosofaríngeo. Éste podría sufrir daños durante la adenoidectomía que se pueden manifestar como roncopatía, disfonía, disfagia, desviaciones del paladar, anestesia de la pared posterior de la faringe, etc. 


\section{b. Tardías}

- Insuficiencia velar: Puede producirse tras una adenoidectomía, habitualmente en pacientes de riesgo, como fisuras palatinas (evidentes y submucosas), desproporción palatofaríngea 0 en algunas malformaciones craneofaciales. Se sospecha en pacientes con rinolalia 0 reflujo faringonasal posterior a la cirugía. Habitualmente es transitoria, pero si persiste más allá de 3-4 semanas se debe indicar rehabilitación posquirúrgica por fonoaudiología. De persistir por más de 3 meses, podría estar indicada la resolución quirúrgica mediante distintos tipos de faringoplastia (colgajos, de aumento, velares, esfinterianas, etc.), aunque habitualmente se espera la resolución espontánea apoyada por fonoaudiología hasta los 6-12 meses antes de decidir una resolución quirúrgica. Existen técnicas que permiten mayor precisión como lo son la adenoidectomía bajo control endoscópico, que permite mayor control del procedimiento sin dañar estructuras vecinas. También se puede realizar adenoidectomía parcial exclusivamente del polo superior, conservando el polo inferior para que permita el adecuado cierre velofaríngeo.

- Estenosis nasofaríngea: Complicación tardía tras adenoidectomía. Se produce por remoción excesiva de tejido faríngeo con cicatrización retráctil, disminuyendo el diámetro de la nasofaringe. En casos severos puede requerir faringoplastía de ampliación.

- Estenosis orofaríngea: Complicación cicatricial de amigdalectomía, en la que se producen adherencias entre la base lingual y los pilares amigdalinos, produciendo estenosis. Puede manejarse con corticoides intralesionales 0 cirugía.

- Recidiva: Se manifiesta como reaparición de los síntomas en pacientes con cirugía incompleta, en los que la adenoides vuelve a experimentar hiperplasia. También es frecuente al intervenir a pequeños menores a 12 meses. En el caso de amigdalectomía, se puede observar recidiva en casos de amigdalectomía intracapsular.

- Modificación de la voz: En general no corresponde a una complicación propiamente tal, ya que no implica un riesgo para el paciente. Se produce tras la modificación de los resonadores faríngeos. A veces se produce un aumento en el tono, que habitualmente es transitorio y cede espontáneamente dentro del primer mes.

\section{EFECTOS DE LA CIRUGÍA}

\section{Síndrome de apnea/hipoapnea obstructiva del sueño (SAHOS) en niños}

En la literatura se habla actualmente de respiración desordenada del sueño (sleep-disordered breathing), como un espectro de trastornos de la respiración durante el sueño, que incluye al ronquido, el síndrome de resistencia respiratoria alta y la apnea/hipopnea obstructiva del sueño; cuyo estudio y manejo (incluyendo el rol de la cirugía adenoamigdalina) es materia de intensa actualización e investigación.

El síndrome de apnea/hipopnea obstructiva del sueño o SAHOS, se define como la obstrucción parcial y prolongada de la vía aérea superior, que interrumpe la ventilación normal durante el sueño con diversas repercusiones sistémicas. Se presenta en aproximadamente el $3 \%$ de pacientes pediátricos, con un peak de incidencia entre los 2 y 8 años. Dentro de las manifestaciones clínicas nocturnas encontramos ronquidos, sueño intranquilo, esfuerzo respiratorio ineficaz, apneas y enuresis. Dentro de las manifestaciones diurnas puede existir rinolalia, dificultad respiratoria, voz gangosa, somnolencia, hiperactividad, alteración del incremento pondoestatural, disminución del rendimiento escolar, problemas conductuales y en casos más severos pueden llegar a presentar corpulmonale. EI SAHOS es una enfermedad multifactorial, y dentro de los principales factores de riesgo podemos destacar la hipertrofia adenoamigdalina, obesidad, anomalías craneofaciales, alteraciones neuromosculares y síndrome de Down.

El gold standard diagnóstico es la polisomnografía, en la que un índice de apnea/hipopnea (IAH) mayor 0 igual a 5 eventos por hora, traduce una alteración ${ }^{17}$. Habitualmente no solicitamos este examen en niños previo a una adenoamigdalectomía, por lo que se tiende a hablar de roncopatía con pausas, si el SAHOS no ha sido demostrado. 
Respecto a sus consecuencias en población pediátrica, más que hipersomnia, en los niños el SAHOS produce principalmente alteraciones cognitivas, mal comportamiento y alteración de la calidad de vida ${ }^{8}$. Se ha comprobado que dentro del control neuroconductual de los niños, la atención y la función ejecutiva son sensibles a la hipoxia intermitente que caracteriza el SAHOS, sin embargo, no está demostrada una mejoría cognitiva significativa de la adenoamigdalectomía versus la vigilancia cercana de estos pacientes. Los parámetros que sí presentan mejorías significativas tras la adenoamigdalectomía temprana versus la vigilancia cercana son las alteraciones conductuales, definidas como inquietud, impulsividad y labilidad emocional, la sintomatología respiratoria, la calidad de vida determinada por el propio paciente según encuestas objetivas y los parámetros polisomnográficos, evidenciándose una mejoría significativa con $79 \%$ en aquellos pacientes sometidos a polisomnografía temprana versus $46 \%$ en aquellos sometidos a vigilancia cercana. No hubo diferencias significativas en los resultados según la edad o el índice de masa corporal (IMC) de los participantes, sin embargo se evidenciaron peores resultados en pacientes de raza negra ${ }^{18}$.

Existe evidencia contradictoria acerca de la relación entre SAHOS e hipertensión en pacientes pediátricos. Se ha visto que los menores con roncopatía y diagnóstico de SAHOS presentan cifras tensionales elevadas respecto a los menores de su edad, aun cuando presenten roncopatía, sin SAHOS asociado. Dentro de las alteraciones encontramos: presión arterial nocturna elevada y disminución del dipping nocturno, ambos valores relacionados con la obesidad y el índice de desaturación de oxigeno del paciente ${ }^{19}$. A pesar que existe la necesidad de mayores estudios respecto al tema, se puede inferir que el SAHOS en pacientes pediátricos, al igual que en pacientes adultos, se convierte en un factor de riesgo cardiovascular aumentando la incidencia de enfermedades crónicas a futuro.

\section{Infecciones adenoamigdalinas}

La evidencia actual muestra efectos beneficiosos de la adenoamigdalectomía en pacientes con cuadros infecciosos severos, que presenten entre 3 a 6 episodios al año, con alteración de la calidad de vida tanto personal como de sus cuidadores. Además disminuye el uso de ATB y el riesgo de complicaciones ${ }^{12,20}$. También se observan efectos beneficiosos de la cirugía en pacientes con sintomatología respiratoria obstructiva severa, secundaria a la hipertrofia adenoamigdalina.

En pacientes con cuadros infecciosos moderados, la cirugía no muestra beneficios respecto a episodios de fiebre por año, infecciones faríngeas por año, infecciones de la vía respiratoria alta y calidad de vida.

La adenoamigdalectomia no tiene mayor beneficio clínico en comparación con la observación, en niños con síntomas leves de infecciones faríngeas e hipertrofia adenoamigdalina

\section{Inmunidad posterior a la cirugía}

La posible disminución de la respuesta inmune posterior a la cirugía es un tema controvertido. Esta teoría, basada en el rol inmune que posee el tejido adenoamigdalino, está muy difundido dentro de la población general. Aún así, tanto la experiencia clínica como los últimos estudios realizados en pacientes pediátricos desmienten esta teoría, demostrando que tanto la inmunidad humoral como la celular se mantienen casi intactas posterior al procedimiento ${ }^{21}$, tanto a corto como a largo plazo $^{22-24}$. Pudiese existir una disminución respecto a los valores preoperatorios en niños con un sistema inmune "exaltado", sometido a amigdalitis/ adenoiditis crónica o debido a la misma hiperplasia de ambas, sin embargo a pesar del descenso, los valores de inmunoglobulinas y células inmunes se mantiene en rangos normales ${ }^{24}$. También se postula la posibilidad de que el resto del sistema inmune asociado a mucosas compense, respondiendo por el tejido extraído. Debido a esto, se concluye que la adenoamigdalectomía no genera un estado de inmunosupresión en pacientes pediátricos ya que sus valores (medidos en inmunoglobulinas, linfocitos $B$ y $T$ ) se mantienen cercanos a los preoperatorios y similares a los de pacientes pediátricos de la misma edad no sometidos a intervención quirúrgica ${ }^{25}$. 


\section{BIBLIOGRAFÍA}

1. López M. Adenoides y amígdalas. Rev Chil Pediatr 2001; 72: 251-5.

2. Kraitrakul S, Sirithunyaporn S, Yimtae K. Distribution of minor salivary glands in the peritonsillar space. J Med Assoc Thai 2001; 84: 371-8.

3. Goldsmith AJ. Tonsillectomy, adenoidectomy and UPPP. En: Bluestone CD, Rosenfeld RM , ed. Surgical Atlas of Pediatric Otolaryngology, 2nd Ed. BC Decker; 2002, p. 379-406.

4. Isaacson G. Pediatric Tonsillectomy: An EvidenceBased Approach. Otolaryngol Clin N Am 2014; 47: 673-90.

5. Messner AH. Tonsillectomy. Oper Tech Otolaryngology 2005; 16: 224-8.

6. Nicollas R, Bonneru JJ, Roman S, Triglia JM. Adenoidectomía. Amigdalectomía. En: Enciclopedia Médico-Quirúrgica - Cirugía otorrinolaringológica y cervicofacial. Paris: Elsevier SAS; 2005 - E - 46-330.

7. Cervera Escario J, Del Castillo Martín F, Gómez Campderá Ja, Gras Albert JR, Pérez Piñero B, Villafruela Sanz Ma. Indicaciones de Adenoidectomía y Amigdalectomía: Documento de Consenso entre la Sociedad Española de Otorrinolaringología y Patología Cervicofacial y la Asociación Española de Pediatría. Acta Otorrinolaringol Esp 2006; 57 : 59-65.

8. Munir N, Clarke R. Indications for tonsillectomy: the evidence base and current UK practice. $\mathrm{Br} \mathrm{J}$ Hosp Med (Lond) 2009; 70: 344-7.

9. Aedo C, Muñoz D. Indicaciones no tradicionales de amigdalectomía. Rev Otorrinolaringol Cir Cabeza Cuello 2010; 70: 165-74.

10. Godoy JM, Godoy A, Godoy JM, Nazar G. Técnicas quirúrgicas actuales en adenoamigdalectomía. Rev Med Clin Condes 2009; 20: 491-9.

11. Bahamonde H, Sepúlveda JC, Olavarría C, Arteaga P, Cruzat A. Estudio comparativo de amigdalectomía con radiofrecuencia versus técnica tradicional. Rev Otorrinolaringol Cir Cabeza Cuello 2004; 64: 113-8.

12. Oomen KP. Evidence-Based Practice: Pediatric Tonsillectomy. Orolaryngol Clin N Am 2012; 45: 1071-81.

13. Hatton RC. Bismuth Subgallate - Epinephrine
Paste in Adenotonsillectomies. Ann Pharmacother 2000; 34: 522-5.

14. Wormald PJ, Sellars SL. Bismuth subgallate: a safe means to a faster adenotonsillectomy. $J$ Laryngol Otol 1994; 108: 761-2.

15. Sørensen WT, Henrichsen J, Bonding P. Does bismuth subgallate have haemostatic effects in tonsillectomy? Clin Otolaryngol Allied Sci 1999; 24: $72-4$.

16. Murray AD, Gibbs SR, Billings KR, Biavati MJ. Respiratory difficulty following bismuth subgallate aspiration. Arch Otolaryngol Head Neck Surg 2000; 126: 79-81.

17. Dal ZY, Huang DY, Zhou CY. Effects of partial tonsillectomy on the immune functions of children with obstructive sleep apnea-hypopnea syndrome at early stage. Genet Mol Res 2014; 13: 3895-902.

18. Marcus CL, Moore RH, Rosen CL, Giordani B, Garetz SL, Taylor HG, et al. Childhood Adenotonsillectomy Trial (CHAT). A randomized trial of adenotonsillectomy for childhood sleep apnea. N Engl J Med 2013; 368: 2366-76.

19. Xu Z, LI B, SHEN K. Ambulatory blood pressure monitoring in Chinese children with obstructive sleep apnea/hypopnea syndrome. Pediatr Pulmonol 2013; 48: 274-9.

20. vanStaalj BK, van den Akker EH, Rovers MM, HordiJk GJ, Hoes AW, SchiLder AG. Effectiveness of adenotonsillectomy in children with mild symptoms of throat infections or adenotonsillar hypertrophy: open, randomised controlled trial. BMJ 2004; 329: 651.

21. Bitar MA, Dowli A, Mourad M. The effect of tonsillectomy on the immune system: A systematic review and meta-analysis. Int $J$ Pediatr Otorhinolaryngol 2015; 79: 1184-91.

22. Kaygusuz I, Alpay HC, Gödekmerdan A, Karlidag T, Keles E, Yalcin S, et al. Evaluation of long-term impacts of tonsillectomy on immune functions of children: a follow-up study. Int J Pediatr Otorhinolaryngol 2009; 73: 445-9.

23. van den Akker EH, Sanders EA, van StaalJ BK, RIJKERS GT, Rovers MM, HoEs AW, et AL. Long-term effects of pediatric adenotonsillectomy on serum immunoglobulin levels: results of a randomized controlled trial. Ann Allergy Asthma Immunol 2006; 97: 251-6. 
24. Santos FP, Weber R, Fortes BC, PIgnatari SS. Short and long term impact of adenotonsillectomy on the immune system. Braz J Otorhinolaryngol 2013; 79: 28-34.
25. IKInCIOGULlarI A, Dogu F, IKIncIogullari A, EgIN Y, Babacan $E$. Is immune system influenced by adenotonsillectomy in children? Int J Pediatr Otorhinolaryngol 2002; 66: 251-7. 